\title{
Tyrosine levels are associated with insulin resistance in patients with nonalcoholic fatty liver disease
}

This article was published in the following Dove Press journal:

Hepatic Medicine: Evidence and Research

3 June 2015

Number of times this article has been viewed

\author{
Miwa Kawanaka' \\ Ken Nishino' \\ Takahito Oka' \\ Noriyo Urata' \\ Jun Nakamura' \\ Mitsuhiko Suehiro' \\ Hirofumi Kawamoto' \\ Yasutaka Chiba ${ }^{2}$ \\ Gotaro Yamada' \\ 'Department of General Internal \\ Medicine 2, Kawasaki Hospital, \\ Kawasaki Medical School, Okayama, \\ Japan; ${ }^{2}$ Clinical Research Center, Kinki \\ University Hospital, Sayama, Japan
}

Correspondence: Miwa Kawanaka Department of General Internal Medicine 2, Kawasaki Hospital, Kawasaki Medical School, 2-I-80 Nakasange, Kita-ku, Okayama City, Okayama 700-8505, Japan

Tel +818622521 II

Fax $+8 \mid 862328343$

Emailm.kawanaka@med.kawasaki-m.ac.jp
Objective: Amino acid imbalance is often found in patients with cirrhosis, and this imbalance is associated with insulin resistance. However, the mechanism underlying the relationship between amino acid imbalance and insulin resistance remains unclear. We evaluated serum amino acid concentrations in patients with nonalcoholic fatty liver disease to determine if any of the levels of amino acids were associated with the biochemical markers and fibrosis stage of nonalcoholic steatohepatitis (NASH).

Methods: In 137 patients with nonalcoholic fatty liver disease who underwent liver biopsy, plasma levels of branched-chain amino acid (BCAA), tyrosine (Tyr), and the BCAA-to-Tyr ratio values were determined using mass spectroscopy. These values were then assessed for associations with fibrosis stage, anthropometric markers (age, sex, and body mass index), biochemical markers (alanine aminotransferase, aspartate aminotransferase, $\gamma$-glutamyl transpeptidase, albumin, platelet count, total cholesterol, triglycerides, low-density lipoprotein cholesterol, and glycosylated hemoglobin), and relevant disease-specific biomarkers (homeostasis model assessment of insulin resistance [HOMA-IR], serum iron, ferritin, leptin, adiponectin, high-sensitivity C-reactive protein, and hyaluronic acid).

Results: Serum albumin levels, plasma BCAA levels, and BCAA-to-Tyr ratio values were negatively associated with the fibrosis stage. In contrast, Tyr levels increased with increasing fibrotic staging. Tyr levels were also correlated with HOMA-IR results.

Conclusion: Plasma BCAA levels in patients with NASH decreased with increasing liver fibrosis, while Tyr levels increased with increasing fibrotic stage. These results suggest that amino acid imbalance and insulin resistance are intimately involved in a complex pathogenic mechanism for NASH.

Keywords: nonalcoholic steatohepatitis, amino acid imbalance, branched-chain amino acids, branched-chain amino acid-to-tyrosine ratio (BTR)

\section{Introduction}

Cirrhosis leading to hepatic encephalopathy is associated with abnormal protein and amino acid metabolism and a decreased Fisher's ratio (the molar ratio of branched-chain amino acids [BCAAs] [leucine, valine, isoleucine] to aromatic amino acids [phenylalanine, tyrosine $\{\mathrm{Tyr}\}]$ ). The prognosis of patients with hepatic encephalopathy is also greatly affected by amino acid imbalance. ${ }^{1,2}$ Furthermore, amino acid imbalance often occurs in cirrhotic patients without hepatic encephalopathy, as well as patients with chronic hepatitis. ${ }^{3}$ Treating these patients with amino acid supplements has been shown to improve both their Fisher's ratio and glucose metabolism. Moreover, studies have suggested a strong association between amino acids, glucose metabolism, and insulin resistance..$^{4-6}$ 
Although insulin resistance is a known cause of nonalcoholic steatohepatitis (NASH), ${ }^{7,8}$ the mechanism underlying the relationship between amino acid imbalance and insulin resistance in NASH is poorly understood. Therefore, the present study aimed to evaluate the association between plasma concentrations of BCAAs, Tyr, and disease factors that are associated with nonalcoholic fatty liver disease (NAFLD) (fibrosis stage, blood biochemical test results, and insulin resistance) in patients who underwent liver biopsy.

\section{Materials and methods Patients}

In total, 137 patients with NASH (67 men, 70 women; mean age, $55.4 \pm 15.4$ years) were enrolled in this study. Fifty-eight patients were diagnosed with stage 0-1 NASH fibrosis, 29 patients with stage 2, 38 patients with stage 3 , and 13 patients with stage 4. All patients underwent a liver biopsy at Kawasaki Hospital, Kawasaki Medical School, Okayama, Japan between 2008 and 2012. All patients with stage 4 NASH had class A Child-Pugh disease. Patients were excluded if they had received amino acid supplementation or were receiving treatment for diabetes. Although $10 \%$ and $20 \%$ of patients were receiving treatment for hypertension and dyslipidemia, respectively, no significant difference in the use of these medications was observed across the fibrosis stages.

This study's protocol was approved by our institutional ethics committee. Informed consent was received from the patients.

\section{Diagnostic criteria}

The NAFLD criteria were as follows: 1) alcohol intake of $\leq 20$ g/week; 2) hepatitis B surface antigen negativity and hepatitis $\mathrm{C}$ antigen negativity, with the exclusion of autoimmune liver disease, drug-induced hepatic disorder, and metabolic liver disease (eg, Wilson's disease and hemochromatosis); and $3)$ the presence of steatosis $(>30 \%)$ or steatohepatitis. The pathological classification was performed using the NASH Clinical Research Network scoring system. ${ }^{9}$

\section{Amino acid measurements}

Fasting blood samples were collected on the day of the liver biopsy. Amino Tag Wako (Wako Pure Chemical Industries, Ltd, Osaka, Japan) was used to measure plasma BCAA levels, Tyr levels, and BCAA-to-Tyr ratio (BTR) for each patient. This method of amino acid analysis uses an amino acid derivative for pretreatment and reaction (Mass Trak ${ }^{\mathrm{TM}}$ AAA Derivatization kit). The column was a Mass Trak ${ }^{\mathrm{TM}}$ amino acid analysis column $(2.1 \times 150 \mathrm{~mm})$, and we used the ACQUITY UPLC system with Empower ${ }^{\mathrm{TM}} 2$ software for data analysis.

The values for each amino acid measurement were then assessed for possible correlations with age and liver disease stage. Correlations were also assessed for alanine aminotransferase (ALT), aspartate aminotransferase (AST), $\gamma$-glutamyl transpeptidase $(\gamma$-GTP), albumin, platelet count, total cholesterol, triglycerides, low-density lipoprotein cholesterol (LDL-C), glycosylated hemoglobin $\left(\mathrm{HbA}_{1 \mathrm{c}}\right)$, homeostasis model assessment of insulin resistance (HOMA-IR), serum iron level, ferritin, leptin, adiponectin, high-sensitivity $\mathrm{C}$-reactive protein (hs-CRP), and hyaluronic acid.

\section{Statistical analysis}

Laboratory characteristics were compared across the stage $0-1$, stage 2 , stage 3 , and stage 4 groups using the KruskalWallis test. For Tyr and BCAA levels, as well as for BTR, all pairs from stage $0-1$, stage 2 , stage 3 , and stage 4 were compared using Scheffe's method to account for the multiplicity of the statistical tests. In addition, we conducted linear regression analyses to explore the factors that affected the Tyr and BCAA levels and the BTR value. First, univariable regression analyses were conducted, and significant factors from the univariable analyses were subsequently included in the multivariable regression analyses as explanatory variables. For all statistical tests, the threshold for significance was defined as a $P$-value of $<0.05$. JMP software (version 9.0.1; SAS Institute Inc., Cary, NC, USA) was used for all statistical analyses.

\section{Results}

Patient demographics and biochemical test results are shown in Tables 1 and 2. Several significant associations were found between the plasma biochemical levels and the patients' liver fibrosis stage. Serum albumin, plasma BCAA, and BTR values all decreased as the fibrosis staging

Table I Fibrotic stages of the subjects and their clinical backgrounds

\begin{tabular}{llllll}
\hline & NASH & & & P-value \\
\cline { 2 - 5 } & Stage 0-I & Stage 2 & Stage 3 & Stage 4 & \\
\hline $\mathrm{n}$ & 58 & 29 & 38 & 13 & \\
Age (years) & $49.8 \pm 14.8$ & $57.1 \pm 14.1$ & $59.3 \pm 15.5$ & $65.4 \pm 11.0$ & 0.004 \\
Male/ & $36 / 22$ & $14 / 15$ & $14 / 24$ & $4 / 9$ & 0.065 \\
female $(\mathrm{n})$ & & & & & \\
BMI $\left(\mathrm{kg} / \mathrm{m}^{2}\right)$ & $26.1 \pm 4.1$ & $27.3 \pm 3.3$ & $30.1 \pm 6.6$ & $27.5 \pm 3.6$ & 0.075 \\
\hline
\end{tabular}

Note: Data are presented as mean \pm standard deviation.

Abbreviations: NASH, nonalcoholic steatohepatitis; BMI, body mass index. 
Table 2 Laboratory characteristics of patients with NASH

\begin{tabular}{|c|c|c|c|c|c|}
\hline & \multicolumn{4}{|l|}{ NASH } & \multirow[t]{2}{*}{$P$-value } \\
\hline & Stage 0-I & Stage 2 & Stage 3 & Stage 4 & \\
\hline ALT (IU/L) & $50.0 \pm 31.7$ & $74.7 \pm 62.6$ & $65.1 \pm 37.3$ & $47 \pm 57.4$ & 0.0302 \\
\hline AST (IU/L) & $31.6 \pm 11.9$ & $51.3 \pm 36.7$ & $50 \pm 24$ & $42.8 \pm 22.8$ & 0.0002 \\
\hline$\gamma$-GTP (IU/L) & $70.5 \pm 64$ & $53.4 \pm 37.3$ & $57.1 \pm 34.6$ & $73.1 \pm 53.1$ & 0.6556 \\
\hline Cholinesterase (U/L) & $361 \pm 98$ & $338 \pm 105$ & $330 \pm 90$ & $298 \pm 93$ & 0.124 \\
\hline Total bilirubin (mg/dL) & $0.9 \pm 0.3$ & $0.8 \pm 0.2$ & $0.9 \pm 0.4$ & $0.9 \pm 0.4$ & 0.8791 \\
\hline Platelets $\left(10^{4} / \mu \mathrm{L}\right)$ & $22.1 \pm 5.9$ & $21.2 \pm 6.8$ & $20.6 \pm 7.1$ & $14.3 \pm 4.5$ & 0.0004 \\
\hline Albumin $(g / d L)$ & $4.6 \pm 0.2$ & $4.4 \pm 0.3$ & $4.3 \pm 0.3$ & $4.1 \pm 0.5$ & $<0.000$ I \\
\hline Total cholesterol (mg/dL) & $193 \pm 38.3$ & $20 \mathrm{I} \pm 28.2$ & $195 \pm 24.8$ & $186 \pm 55.2$ & 0.6063 \\
\hline Triglyceride (mg/dL) & $155 \pm 84.9$ & $142.2 \pm 58.7$ & $147.5 \pm 77.8$ & $103.6 \pm 26.4$ & 0.199 \\
\hline HDL-C (mg/dL) & $48.5 \pm \mathrm{II} .2$ & $43.5 \pm 9.2$ & $47.7 \pm 18.1$ & $46.9 \pm 13$ & 0.3756 \\
\hline LDL-C (mg/dL) & $114.9 \pm 34.4$ & $\mid 29.4 \pm 24.1$ & $118.5 \pm 27.3$ & $118.7 \pm 52.7$ & 0.2545 \\
\hline Serum iron $(\mu g / d L)$ & $12 \mid \pm 32$ & $110 \pm 38$ & $115 \pm 37$ & $132 \pm 56$ & 0.3812 \\
\hline Ferritin $(\mathrm{ng} / \mathrm{mL})$ & $143 \pm 95$ & $144 \pm 128$ & $168 \pm 154$ & $202 \pm 323$ & 0.9584 \\
\hline Hyaluronic acid (ng/mL) & $36.8 \pm 25.1$ & $55 \pm 58$ & $76 \pm 108$ & $168 \pm 142$ & 0.0002 \\
\hline P-III-P (U/mL) & $0.56 \pm 0.12$ & $0.6 \pm 0.2$ & $0.75 \pm 0.5$ & $0.7 \pm 0.18$ & 0.0146 \\
\hline Type IV collagen $7 \mathrm{~S}(\mathrm{ng} / \mathrm{mL})$ & $3.6 \pm 0.6$ & $4.0 \pm 0.9$ & $4.7 \pm 1.3$ & $5.6 \pm 1.5$ & $<0.0001$ \\
\hline hs-CRP (mg/dL) & $152 \pm 213$ & $197 \pm 213$ & $222 \pm 224$ & $145 \pm 102$ & 0.3092 \\
\hline HOMA-IR & $2.7 \pm 2$ & $3.5 \pm 2.5$ & $3.6 \pm 1.6$ & $3.4 \pm 1.6$ & 0.0034 \\
\hline Leptin (ng/mL) & $8.3 \pm 5.5$ & $12.5 \pm 11.4$ & $17.1 \pm 12.2$ & $12.3 \pm 9.6$ & 0.0018 \\
\hline Adiponectin $(\mu g / m L)$ & $7.0 \pm 3.9$ & $7.3 \pm 3.8$ & $6.1 \pm 2.6$ & $7.4 \pm 3.8$ & 0.7889 \\
\hline
\end{tabular}

Note: Data are presented as mean \pm standard deviation.

Abbreviations: NASH, nonalcoholic steatohepatitis; ALT, alanine aminotransferase; AST, aspartate aminotransferase; $\gamma$-GTP, $\gamma$-glutamyl transpeptidase; HDL-C, highdensity lipoprotein cholesterol; LDL-C, low-density lipoprotein cholesterol; P-III-P, type III procollagen-N-peptide; hs-CRP, high-sensitivity C-reactive protein; HOMA-IR, homeostasis model assessment of insulin resistance.

increased. The mean serum albumin level at each fibrosis stage was $4.8 \mathrm{~g} / \mathrm{dL}$ in stage $0-1,4.6 \mathrm{~g} / \mathrm{dL}$ in stage $2,4.3$ $\mathrm{g} / \mathrm{dL}$ in stage 3 , and $4.0 \mathrm{~g} / \mathrm{dL}$ in stage 4 (stage $0-1$ versus stage $3, P<0.01$; stage $0-1$ versus stage $4, P<0.001$ ) (Figure 1). The mean plasma BCAA level at each fibrosis stage was $516.4 \mu \mathrm{mol} / \mathrm{L}$ in stage $0-1,514.6 \mu \mathrm{mol} / \mathrm{L}$ in stage 2, $494.8 \mu \mathrm{mol} / \mathrm{L}$ in stage 3 , and $463.1 \mu \mathrm{mol} / \mathrm{L}$ in stage 4 (stage
0-1 versus stage 4, $P<0.01$ ) (Figure 2). However, a small number of patients exhibited plasma BCAA levels that were lower than the reference level. The mean BTR value at each fibrosis stage was 6.8 in stage $0-1,6.8$ in stage 2 , 5.9 in stage 3 , and 5.0 in stage 4 (stage $0-1$ versus stage 3 , $P<0.01$; stage 2 versus stage $3, P<0.01$; stage $0-1$ versus stage $4, P<0.001$ ) (Figure 3 ). In contrast, the Tyr levels

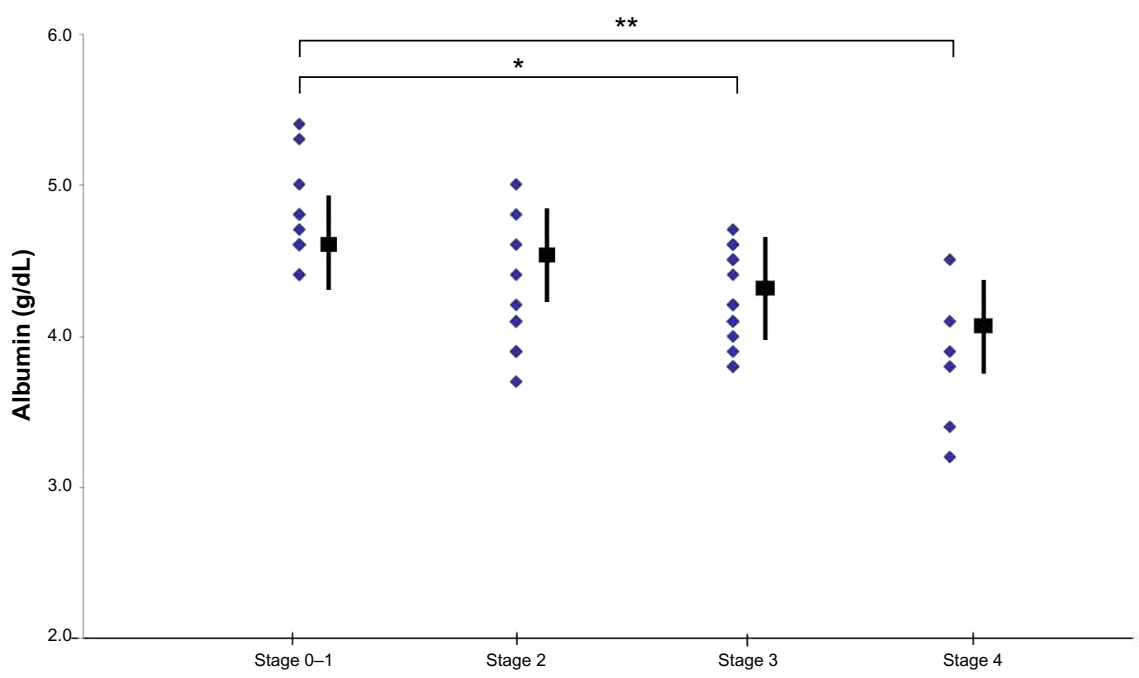

Figure I Changes in albumin levels according to the progression of fibrosis.

Notes: *Stage $0-1$ versus stage $3(P<0.01)$; **stage $0-I$ versus stage $4(P<0.00 I)$. The shaded blue area indicates bases of Albumin, the black box and line indicates data are presented as mean \pm standard deviation. 


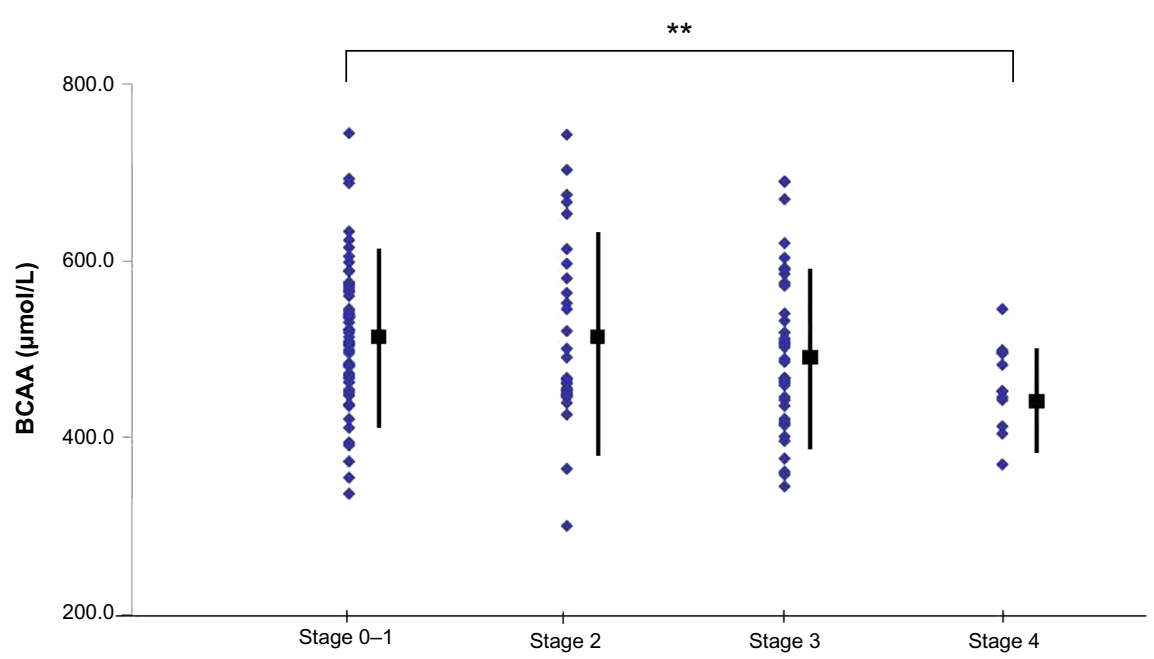

Figure 2 Changes in branched-chain amino acid (BCAA) levels according to the progression of fibrosis.

Note: **Stage $0-1$ versus stage $4(P<0.01)$.

tended to increase at each fibrosis stage: $77.1 \mu \mathrm{mol} / \mathrm{L}$ in stage $0-1,76.1 \mu \mathrm{mol} / \mathrm{L}$ in stage $2,84.1 \mu \mathrm{mol} / \mathrm{L}$ in stage 3 , and $99.9 \mu \mathrm{mol} / \mathrm{L}$ in stage 4 (stage $0-1$ versus stage 4 , $P<0.01$; stage 2 versus stage $3, P<0.05$ ) (Figure 4).

Univariable analysis revealed that the Tyr levels were significantly correlated with HOMA-IR, platelet, albumin, leptin, LDL-C, and hyaluronic acid levels. Multivariable analysis subsequently revealed that the Tyr levels were correlated with HOMA-IR values (Tables 3 and 4). Univariable analysis also revealed that the plasma BCAA levels were significantly correlated with adiponectin, albumin, and hyaluronic acid levels, although no correlations with the plasma BCAA levels were observed in the multivariable analysis (Tables 5 and 6).

\section{Discussion}

Amino acids are one of the molecular substrates that are used for gluconeogenesis, ${ }^{10}$ and the liver and skeletal muscles serve as the primary sites of amino acid metabolism. In addition, BCAAs are metabolized in the brain and skeletal muscles, where they produce alanine and glutamine as intermediate metabolites; the alanine and glutamine are then further metabolized in the kidneys and small intestine. Aromatic amino acids and methionine are primarily metabolized in the liver, and are then transported by the glucose-alanine shuttle between the liver and skeletal muscles to produce glucose.

In patients with liver cirrhosis, especially those with hepatic encephalopathy, amino acid imbalance occurs as BCAA levels decrease and aromatic amino acid levels

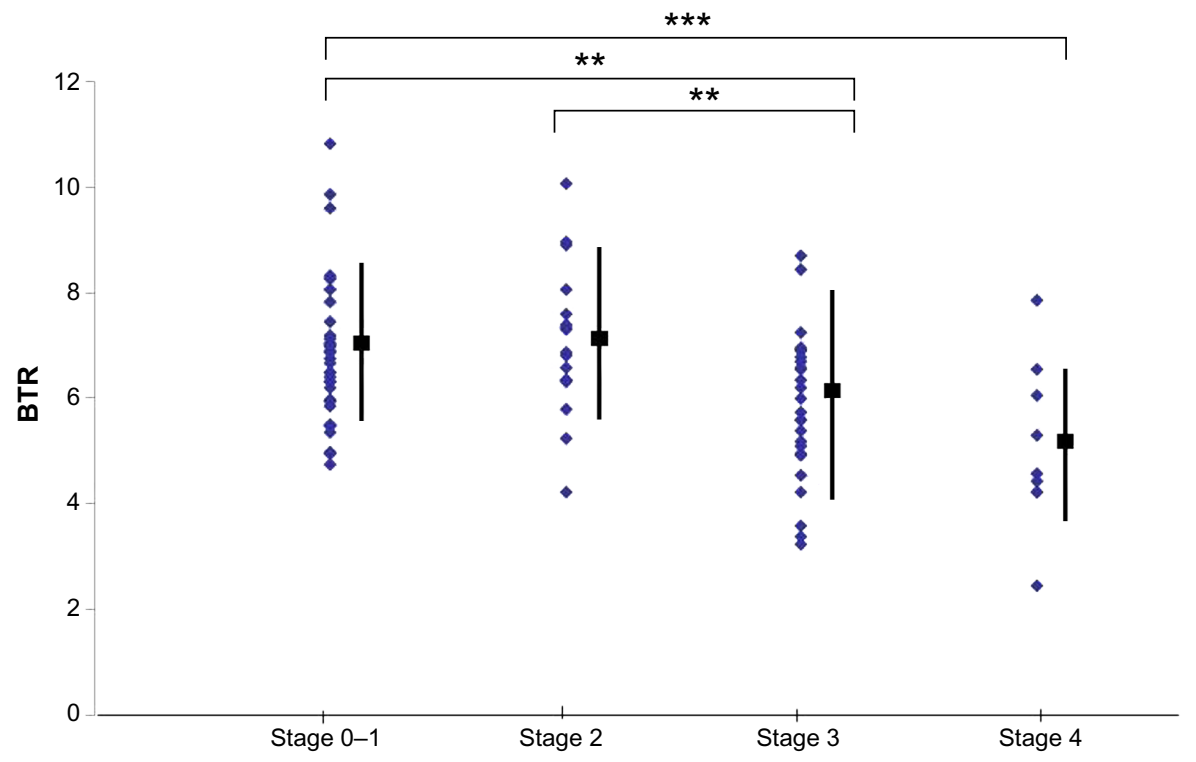

Figure 3 Changes in the branched-chain amino acid-to-tyrosine ratio (BTR) according to the progression of fibrosis. Notes: ** Stage $0-I$ versus stage 3 , stage 2 versus stage $3(P<0.0 I)$; ****stage $0-I$ versus stage $4(P<0.00 I)$. 


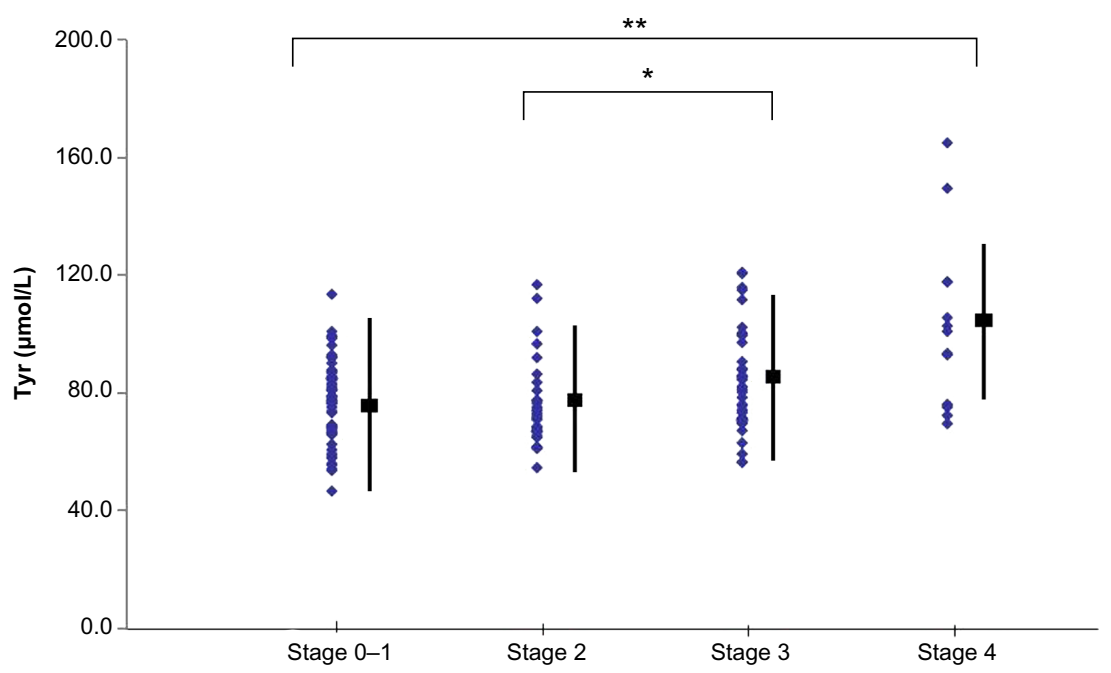

Figure 4 Changes in tyrosine (Tyr) levels according to the progression of fibrosis. Notes: **Stage $0-I$ versus stage $4(P<0.01)$; *stage 2 versus stage $3(P<0.05)$.

increase (resulting in a decreased Fisher's ratio). ${ }^{1-3}$ This decrease in BCAA levels may be due to their consumption by skeletal muscles for ammonia detoxification and generating metabolic energy. ${ }^{11}$ The molecular mechanism underlying this decrease is believed to result from the increased production of tumor necrosis factor-alpha ( $\mathrm{TNF} \alpha$ ), which causes bind-

Table 3 Univariable analysis of factors associated with tyrosine in patients with nonalcoholic steatohepatitis

\begin{tabular}{lllll}
\hline & Estimate & $\mathbf{9 5 \%} \mathbf{C l}$ & & $P$-value \\
\hline Age & $0.197 \mathrm{I}$ & -0.0125 & 0.4066 & $0.065 \mathrm{I}$ \\
ALT & -0.0318 & -0.1022 & 0.0385 & 0.3726 \\
AST & 0.0244 & -0.1023 & 0.1510 & $0.704 \mathrm{I}$ \\
$\gamma$-GTP & 0.0036 & $-0.058 \mathrm{I}$ & 0.0654 & 0.9076 \\
Cholinesterase & 0.0028 & -0.0300 & 0.0356 & 0.8652 \\
Platelets & $-0.86 \mathrm{II}$ & -1.3093 & -0.4130 & 0.0002 \\
Albumin & -12.2445 & -20.2752 & -4.2138 & $0.003 \mathrm{I}$ \\
Total cholesterol & -0.0649 & $-0.157 \mathrm{I}$ & 0.0272 & 0.1657 \\
Triglyceride & 0.0027 & $-0.040 \mathrm{I}$ & 0.0456 & 0.9000 \\
HDL-C & 0.1278 & -0.1063 & 0.3619 & 0.2821 \\
LDL-C & -0.1076 & -0.2019 & -0.0132 & 0.0257 \\
Serum iron & 0.0596 & -0.0254 & 0.1447 & 0.1679 \\
Ferritin & -0.0005 & -0.0218 & 0.0208 & 0.9630 \\
Hyaluronic acid & 0.0935 & 0.0632 & 0.1239 & $<0.000 \mathrm{I}$ \\
P-III-P & $5.468 \mathrm{I}$ & -4.0928 & $15.029 \mathrm{I}$ & 0.2599 \\
Type IV collagen $7 \mathrm{~S}$ & 7.1364 & 5.3569 & 8.9159 & $<0.000 \mathrm{I}$ \\
hs-CRP & 9.9526 & -6.0927 & 25.9979 & 0.2216 \\
HOMA-IR & 0.4952 & 0.0909 & 0.8994 & 0.0168 \\
Leptin & 0.4612 & 0.1604 & 0.7621 & 0.0030 \\
Adiponectin & 0.8847 & -0.2465 & 2.0158 & 0.1236 \\
\hline
\end{tabular}

Abbreviations: $\mathrm{Cl}$, confidence interval; ALT, alanine aminotransferase; AST, aspartate aminotransferase; $\gamma$-GTP, $\gamma$-glutamyl transpeptidase; HDL-C, high-density lipoprotein cholesterol; LDL-C, low-density lipoprotein cholesterol; hs-CRP, highsensitivity C-reactive protein; P-III-P, type III procollagen-N-peptide; HOMA-IR, homeostasis model assessment of insulin resistance. ing between the branched-chain $\alpha$-ketoacid dehydrogenase kinase (BDK) and branched-chain $\alpha$-ketoacid dehydrogenase $(\mathrm{BDKDH})$, and results in the inhibition of BDKDH activation. This inhibition in turn causes an increase in the level of activated BDKDH, which enhances the metabolism of BCAAs. ${ }^{12-14}$

In this study, albumin and BCAA levels decreased as the liver fibrosis stage progressed. However, plasma BCAA levels increased in patients with NAFLD in this study. The increase in BCAA levels was proportional to the degree of obesity, which may be related to the decreased activity of mitochondrial branched-chain aminotransferase and BDKDH, as has previously been demonstrated. ${ }^{15}$ In NAFLD patients with insulin resistance, BCAAs may not be metabolized, and their concentration may increase because of the inhibition of BDKDH activity. ${ }^{16}$ Furthermore, Lynch and Adams have reported that a hypothetical mechanism linking increased levels of BCAAs and type 2 diabetes mellitus involves leucine-mediated activation of the mammalian target of rapamycin complex, which results in the uncoupling of insulin signaling at an early stage. ${ }^{17}$

Table 4 Multivariable analysis of factors associated with tyrosine in patients nonalcoholic steatohepatitis

\begin{tabular}{lllll}
\hline & Estimate & $\mathbf{9 5 \%} \mathbf{~ C l}$ & & $P$-value \\
\hline Leptin & 0.0628 & $-0.240 I$ & 0.3657 & 0.6817 \\
Type IV collagen 7S & 4.7077 & 2.1144 & 7.3010 & 0.0005 \\
Hyaluronic acid & 0.0334 & $-0.011 \mathrm{I}$ & 0.0778 & 0.1393 \\
Albumin & -1.2262 & -9.4245 & 6.9722 & 0.7673 \\
Platelets & -0.3382 & -0.7642 & 0.0879 & 0.1185 \\
\hline
\end{tabular}


Table 5 Univariable analysis of factors associated with branchedchain amino acid in patients with nonalcoholic fatty liver and nonalcoholic steatohepatitis

\begin{tabular}{lllll}
\hline & Estimate & $\mathbf{9 5 \%} \mathbf{C l}$ & & $P$-value \\
\hline Age & -1.3778 & -2.3590 & -0.3965 & 0.0063 \\
ALT & 0.3083 & -0.0234 & 0.6400 & 0.0682 \\
AST & -0.0533 & -0.6564 & 0.5497 & 0.8615 \\
$\gamma$-GTP & 0.2045 & -0.0874 & 0.4963 & $0.168 I$ \\
Cholinesterase & 0.2210 & 0.0703 & 0.3717 & 0.0044 \\
Platelets & -0.5748 & -2.8178 & 1.6682 & 0.6131 \\
Albumin & 41.1674 & 2.2967 & 80.0381 & $0.038 I$ \\
Total cholesterol & 0.0418 & -0.3992 & 0.4828 & 0.8514 \\
Triglyceride & 0.3318 & 0.1365 & 0.5270 & 0.0010 \\
HDL-C & -0.8422 & -1.9526 & 0.2642 & 0.1343 \\
LDL-C & 0.0692 & -0.3870 & 0.5253 & 0.7647 \\
Serum iron & 0.2947 & -0.1461 & 0.6454 & 0.2142 \\
Ferritin & 0.0791 & -0.0188 & 0.1770 & 0.1125 \\
Hyaluronic acid & -0.1716 & -0.3262 & -0.0170 & 0.0299 \\
P-III-P & -6.4734 & -50.9725 & 38.0256 & 0.7739 \\
Type IV collagen $7 S$ & -2.3178 & -12.3948 & 7.7593 & 0.6498 \\
hs-CRP & -11.6535 & -89.5940 & 66.2870 & 0.7676 \\
HOMA-IR & -0.9909 & -2.9132 & 0.9314 & 0.3098 \\
Leptin & -0.6307 & -2.0914 & 0.8300 & 0.3943 \\
Adiponectin & -6.5403 & -11.4618 & -1.6187 & 0.0098 \\
\hline
\end{tabular}

Abbreviations: $\mathrm{Cl}$, confidence interval; ALT, alanine aminotransferase; AST, aspartate aminotransferase; $\gamma$-GTP, $\gamma$-glutamyl transpeptidase; HDL-C, high-density lipoprotein cholesterol; LDL-C, low-density lipoprotein cholesterol; hs-CRP, highsensitivity C-reactive protein; P-III-P, type III procollagen-N-peptide; HOMA-IR, homeostasis model assessment of insulin resistance.

Cirrhosis is also associated with decreased insulin clearance, shunt formation, and poor glucose uptake in the liver, which can result in insulin resistance and abnormal glucose metabolism. ${ }^{18,19}$ Furthermore, insulin sensitivity and abnormal glucose tolerance can be predicted in normoglycemic patients, based on their serum levels of BCAAs (leucine, isoleucine, and valine) and aromatic amino acids (phenylalanine and Tyr). ${ }^{19}$ This finding suggests that Tyr is correlated with insulin resistance. In addition, Würtz et al have reported that increased abdominal circumference in healthy men was associated with increased levels of BCAAs, Tyr, and alanine, as well as with decreased levels of glutamine. ${ }^{20}$

Table 6 Multivariable analysis of factors associated with branched chain in patients with nonalcoholic steatohepatitis

\begin{tabular}{lllll}
\hline & Estimate & $\mathbf{9 5 \%} \mathbf{C l}$ & P-value & \\
\hline Age & -0.4498 & -1.9015 & 1.0018 & 0.5387 \\
Adiponectin & -4.5873 & -10.1868 & 1.0122 & 0.1068 \\
Hyaluronic acid & -0.0833 & -0.3105 & 0.1438 & 0.4668 \\
Albumin & -1.3078 & -52.1418 & 49.5262 & 0.9592 \\
Cholinesterase & 0.1727 & -0.0149 & 0.3602 & 0.0706 \\
Triglyceride & 0.0182 & -0.3072 & 0.3437 & 0.9113 \\
\hline
\end{tabular}

Recent studies have also reported that BCAA supplementation reduces adipocyte size, while increasing the expression of peroxisome proliferator-activated receptor- $\alpha$, peroxisome proliferator-activated receptor- $\gamma$, and adiponectin mRNA. ${ }^{21}$ Further studies are needed to reexamine these conflicting reports based on our new findings.

It is unclear whether elevated plasma BCAA level in patients with insulin resistance and abnormal glucose tolerance is a cause of, or a consequence of, liver fibrosis. In rats that were fed a BCAA-fortified high-fat diet, insulin resistance occurred after the activation of the mammalian target of rapamycin via BCAA supplementation, which worked through a serine phosphorylation mechanism. ${ }^{22}$ However, insulin resistance is not enhanced in patients with type 2 diabetes who are treated with leucine. ${ }^{23}$ Therefore, the rodent model may not be broadly applicable to human insulin resistance. Nevertheless, recent studies have reported an improvement in insulin resistance among patients with hepatitis $\mathrm{C}$ cirrhosis who were treated with BCAAs. ${ }^{24,25}$

Serum albumin, Tyr, and BTR values all changed with increasing fibrosis stage, and similar findings have been reported in other types of chronic liver disease..$^{10,26}$ Furthermore, increased Tyr levels were also noted in patients with low fibrosis stages and insulin resistance. Regarding a possible mechanism for the association between Tyr and HOMA-IR levels, insulin resistance increases the levels of $\alpha$-ketobutyrate, which is involved in methionine degradation, and this could lead to an increase in Tyr levels via the subsequent elevation of cysteine levels and decrease in Tyr aminotransferase levels.

Although no direct comparison was made in this study, the BCAA and Tyr profiles in patients with NASH may differ from that in patients with other types of chronic liver disease, such as hepatitis $\mathrm{C}$ or alcoholic liver disease. ${ }^{10}$ Because of the complicated involvement of fibrosis progression, as well as insulin resistance, in the amino acid metabolism of patients with NASH, amino acid imbalance should be considered separately for different liver diseases. In addition, NASH patients without liver cirrhosis and with amino acid imbalances may develop future abnormalities in glucose metabolism.

\section{Conclusion}

Plasma BCAA levels in patients with NASH decreased with increasing liver fibrosis, similar to the findings in other types of chronic liver disease. In contrast, Tyr levels increased with increasing fibrotic stage. These results suggest that amino 
acid imbalance and insulin resistance are intimately involved in a complex pathogenic mechanism for NASH. However, the exact mechanism underlying this relationship has yet to be clarified. To understand the mechanism of amino acid imbalance in NASH, these patients should be monitored closely for glucose intolerance.

\section{Disclosure}

The authors report no conflicts of interest in this work.

\section{References}

1. Tajika M, Kato M, Mohri H, et al. Prognostic value of energy metabolism in patients with viral liver cirrhosis. Nutrition. 2002;18(3):229-234.

2. Moriwaki H, Miwa Y, Tajika M, Kato M, Fukushima H, Shiraki M. Branched-chain amino acids as a protein- and energy-source in liver cirrhosis. Biochem Biophys Res Commun. 2004;313(2):405-409.

3. Morgan MY, Marshall AW, Milsom JP, Sherlock S. Plasma amino-acid patterns in liver disease. Gut. 1982;23(5):362-370.

4. Kawaguchi T, Yamagishi S, Sata M. Branched-chain amino acids and pigment epithelium-derived factor: novel therapeutic agents for hepatitis c virus-associated insulin resistance. Curr Med Chem. 2009;16(36):4843-4857.

5. Urata Y, Okita K, Korenaga K, Uchida K, Yamasaki T, Sakaida I. The effect of supplementation with branched-chain amino acids in patients with liver cirrhosis. Hepatol Res. 2007;37(7):510-516.

6. Nishitani S, Takehana K, Fujitani S, Sonaka I. Branched-chain amino acids improve glucose metabolism in rats with liver cirrhosis. Am J Physiol Gastrointest Liver Physiol. 2005;288(6):G1292-G1300.

7. Pagano G, Pacini G, Musso G, et al. Nonalcoholic steatohepatitis, insulin resistance, and metabolic syndrome: further evidence for an etiologic association. Hepatology. 2003;37(5):1202-1219.

8. Neuschwander-Tetri BA, Caldwell SH. Nonalcoholic steatohepatitis: summary of an AASLD Single Topic Conference. Hepatology. 2003; 37(5):1202-1219.

9. Kleiner DE, Brunt EM, Van Natta M, et al; Nonalcoholic Steatohepatitis Clinical Research Network. Design and validation of a histological scoring system for nonalcoholic fatty liver disease. Hepatology. 2005; 41(6):1313-1321.

10. Michitaka K, Hiraoka A, Kume M, et al. Amino acid imbalance in patients with chronic liver diseases. Hepatol Res. 2010;40(4):393-398.

11. Ichihara A, Koyama E. Transaminase of branched chain amino acids. I. Branched chain amino acids-alpha-ketoglutarate transaminase. J Biochem. 1966;59(2):160-169.
12. Kato M, Miwa Y, Tajika M, Hiraoka T, Muto Y, Moriwaki H. Preferential use of branched-chain amino acids as an energy substrate in patients with liver cirrhosis. Intern Med. 1998;37(5):429-434.

13. Honda T, Fukuda N, Nakano I, et al. Effects of liver failure on branched-chain alpha-keto acid dehydrogenase complex in rat liver and muscle: comparison between acute and chronic liver failure. $J$ Hepatol. 2004;40(3):439-445.

14. Shimomura Y, Honda T, Shiraki M, et al. Branched-chain amino acid catabolism in exercise and liver disease. $J$ Nutr. 2006;136(1 Suppl): 250S-253S

15. She P, Van Horn C, Reid T, Hutson SM, Cooney RN, Lynch CJ. Obesity-related elevations in plasma leucine are associated with alterations in enzymes involved in branched-chain amino acid metabolism. Am J Physiol Endocrinol Metab. 2007;293(6):E1552-E1563.

16. Adams SH. Emerging perspectives on essential amino acid metabolism in obesity and the insulin-resistant state. Adv Nutr. 2011;2(6):445-456.

17. Lynch CJ, Adams SH. Branched-chain amino acids in metabolic signalling and insulin resistance. Nat Rev Endocrinol. 2014:10(12): 723-736.

18. Petrides AS, DeFronzo RA. Glucose and insulin metabolism in cirrhosis J Hepatol. 1989;8(1):107-114.

19. Imano E, Kanda $T$, Nakatani $Y$, et al. Impaired splanchnic and peripheral glucose uptake in liver cirrhosis. J Hepatol. 1999;31(3):469-473.

20. Würtz P, Tiainen M, Mäkinen VP, et al. Circulating metabolite predictors of glycemia in middle-aged men and women. Diabetes Care. 2012;35(8):1749-1756.

21. Terakura D, Shimizu M, Iwasa J, et al. Preventive effects of branchedchain amino acid supplementation on the spontaneous development of hepatic preneoplastic lesions in C57BL/KsJ-db/db obese mice. Carcinogenesis. 2012;33(12):2499-2506.

22. Newgard CB, An J, Bain JR, et al. A branched-chain amino acid-related metabolic signature that differentiates obese and lean humans and contributes to insulin resistance. Cell Metab. 2009;9(4):311-326.

23. Leenders M, Verdijk LB, van der Hoeven L, et al. Prolonged leucine supplementation does not augment muscle mass or affect glycemic control in elderly type 2 diabetic men. $J$ Nutr. 2011;141(6):1070-1076.

24. Ikehara O, Kawasaki N, Maezono K, Komatsu M, Konishi A. Acute and chronic treatment of L-isoleucine ameliorates glucose metabolism in glucose-intolerant and diabetic mice. Biol Pharm Bull. 2008;31(3):469-472.

25. Miyake T, Abe M, Furukawa S, et al. Long-term branched-chain amino acid supplementation improves glucose tolerance in patients with nonalcoholic steatohepatitis-related cirrhosis. Intern Med. 2012;51(16): 2151-2155.

26. Mori T, Ohta M, Sakai M, et al. [Determination of BTR on patients with liver cirrhosis and its clinical usefulness.] Kanzo. 1998;39(5):325-330. Japanese.
Hepatic Medicine: Evidence and Research

\section{Publish your work in this journal}

Hepatic Medicine: Evidence and Research is an international, peerreviewed, open access journal covering all aspects of adult and pediatric hepatology in the clinic and laboratory including the following topics: Pathology, pathophysiology of hepatic disease; Investigation and treatment of hepatic disease; Pharmacology of drugs used for

\section{Dovepress}

the treatment of hepatic disease. Issues of patient safety and quality of care will also be considered. The manuscript management system is completely online and includes a very quick and fair peer-review system, which is all easy to use. Visit http://www.dovepress.com/ testimonials.php to read real quotes from published authors. 